\title{
Diseño y validación de una escala para intereses propedéuticos en México
}

\section{Design and Validation of a Scale for Propaedeutic Interests in Mexico}

\section{Projeto e validação de uma escala para interesses pediátricos no México}

\author{
Abraham Manuel Ortiz-Barradas \\ Universidad Veracruzana, México \\ abrortiz@uv.mx \\ https://orcid.org/0000-0002-3542-9611
}

\section{Resumen}

Con el objetivo de diseñar y validar una escala de tipo Likert para la valoración de los intereses propedéuticos de los estudiantes del sistema de bachillerato general en México, se realizó un estudio en donde participaron 56 voluntarios distribuidos en tres grupos. El primero, con el propósito de establecer el juicio de expertos, se compuso de seis profesionistas de la educación con experiencia en el ámbito de la evaluación y la orientación vocacional; el segundo, con la finalidad de establecer la aplicación de una prueba piloto, estuvo integrado por 10 estudiantes de nivel bachillerato, y el último grupo, para la obtención de resultados, por 40 alumnos también de bachillerato. La escala aplicada se construyó con base en los lineamientos de los programas de estudio expresados en los documentos publicados por la Secretaría de Educación Pública (SEP) vigentes al año 2017. Los resultados se analizaron con programas estadísticos y se obtuvo un alfa de Cronbach de 0.870 , lo que indica una estabilidad y consistencia fiable de la escala.

Palabras clave: área propedéutica, intereses propedéuticos en México, orientación educativa. 


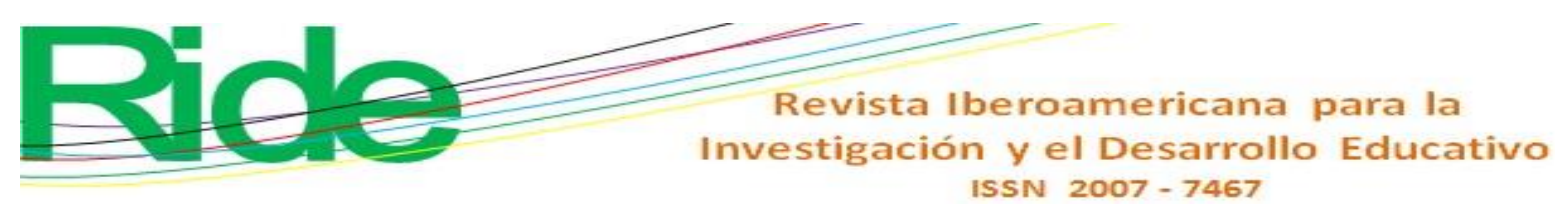

\section{Introducción}

En términos generales, educar se constituye como la actividad que incluye el diseño, la secuenciación y el desarrollo de los criterios de desempeño que convencionalmente se consideran pertinentes y adecuados para que un individuo se adapte y sea productivo en una sociedad.

Dentro del marco de la educación formal, es decir, la educación con parámetros institucionales (Trilla, 1988; Turbay, 2000; Lebrún, 2015; Gómez, 2017), esta tarea supone actividades desde diferentes ámbitos académicos en los que están involucradas las disciplinas de la física, química, biológica, psicológica, antropológica y social — desde sus distintas aristas como la sociología, economía y ciencia política, entre otras.

En su versión más honesta, la misión fundamental de la educación es la de lograr que el aprendiz modifique sus comportamientos con el fin de satisfacer los criterios de conocimiento que la sociedad demanda para así llegar a ser un individuo pleno. Esto genera la necesidad de estudiar cuáles son aquellas condiciones necesarias y suficientes para el logro de este propósito, es decir, para el logro del aprendizaje.

Bajo esta consideración es que la psicología se ha posicionado como una disciplina sine qua non de la educación, en el sentido de que esta se ha apropiado del área del aprendizaje y la ha incluido en su repertorio de categorías propias de su campo de investigación, a partir de lo cual ha tenido una gran influencia sobre contextos escolares y ha generado tendencias educativas tales como la conductista, constructivista, cognitiva y social, entre otras.

En este contexto, uno de los campos de interés ha sido el de la orientación, que se ha desarrollado desde diferentes puntos de vista a partir de modelos mecanicistas, organicistas, contextualistas y de epigénesis probabilística (Sánchez y Valdés, 2003). Modelos que inadvertidamente generan descripciones cuyos sustentos contienen dos problemas generales: 1) utilizar metáforas mentalistas en sus descripciones y 2) recurrir a cruzas de especies para explicar sus supuestos (Ryle, 1949).

La orientación es una actividad cuyo propósito consiste en el desarrollo personal y social del individuo, y a través de la cual se promueve la autoestima, las relaciones humanas y se proporciona atención a las necesidades e intereses de este, del sujeto, de acuerdo con sus potencialidades, habilidades y destrezas (Molina, 2004). En el ámbito educativo, se trata de la atención hacia el estudiante para resolver problemas relacionados con su vida académica. 


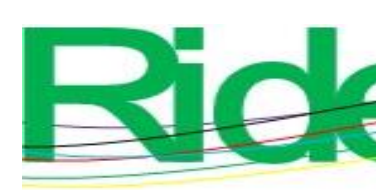

Revista Iberoamericana para la
Investigación y el Desarrollo Educativo
ISSN $2007-7467$

En México, la orientación surge como un servicio docente encaminado hacia la correcta elección de carreras profesionales por parte de los alumnos, y cuyos responsables son los profesores de las diversas escuelas de nivel medio y medio superior.

Aun cuando existen documentos que puntualizan la importancia de la orientación en la vida escolar del estudiante, se distinguen dos problemas iniciales: 1) no existe investigación abstracta de alcance comprensivo y 2) nunca se establecen estrategias de acción pertinentes a tal investigación para dicha actividad.

Esto ha generado que cada orientador opere bajo sus propios criterios y que, muchas veces, aún en el supuesto de que estos estén sustentados por investigación y desarrollo, no cuentan con instrumentos propios que evalúen bajo las características y el contexto de nuestro país y su sistema educativo.

Específicamente para el caso de la educación media superior mexicana, existen dos etapas en donde la orientación juega un papel fundamental, que consisten en la elección del área propedéutica y de carrera profesional.

Para el primer caso, el estudiante que transita del cuarto al quinto semestre de nivel bachillerato debe elegir entre un distinto número de opciones propedéuticas, las cuales tienen como objetivo estimular en el alumno las competencias pertinentes para cada campo disciplinar y así pueda alcanzar una preparación y un desarrollo ideales en su vida académica superior.

El segundo caso consiste en la elección de una carrera profesional que, sustentada en una preparación inicial propedéutica, es una preferencia más específica acerca del núcleo disciplinar que regirá su vida tanto académica como profesional.

En ninguno de los dos casos mencionados existen documentos que dirijan cursos de acción bajo sustentos teóricos psicológicos, su aplicación e instrumentos pertinentes para llevar a cabo orientación educativa en México. Los documentos básicos únicamente prescriben el uso de test vocacionales para el ejercicio de la orientación, pero no establecen la estrategia metodológica en cuanto a qué teoría debe sustentar dicha actividad y cuáles son las técnicas e instrumentos ideales para ello.

Los documentos existentes están orientados más hacia la información de los lineamientos generales sobre las acciones que deberán llevar los responsables de orientación vocacional que a los procedimientos específicos y fundamentos teóricos de un programa de orientación como tal.

Para el caso específico de los estudiantes de cuarto semestre del nivel de bachillerato hasta el momento la literatura pertinente no reporta investigaciones encaminadas hacia este respecto, en 


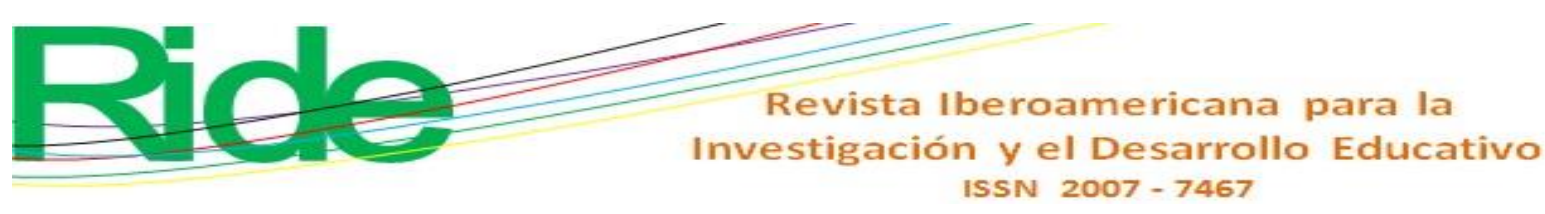

tanto que los estudios solo se circunscriben a sistemas clasificatorios de la orientación y a la elección de carrera profesional. Además, los documentos que ofrece la Secretaría de Educación Pública (SEP) no especifican la labor metodológica del profesional orientador en cuanto a la evaluación, análisis, presentación de resultados e información pertinente necesaria para que el estudiante realice una elección fundamentada y motivada.

En primera instancia, se argumenta que no existe en nuestro país un instrumento que evalúe las aptitudes e intereses de los estudiantes próximos a elegir un área propedéutica conforme a los programas de estudio pertinentes a su contexto social. La SEP, aun cuando presenta en sus documentos básicos la inclusión de la labor profesional de orientación vocacional y educativa, no contempla la precisión de instrumentos, delimitación conceptual y metodológica de dicha práctica.

En los documentos Lineamientos de orientación educativa (SEP, 2010) y Programa de orientación educativa (SEP, 2012a) se hace mención a la actividad de aplicación e interpretación de test vocacionales y baterías psicológicas; sin embargo, no se especifican cuáles han de utilizarse ni las formas de aplicación e interpretación.

Dentro del Manual para el orientador (SEP, 2012b) se establecen cuatro áreas de trabajo bajo las cuales el orientador debe dirigir sus actividades educativas: institucional, escolar, vocacional y psicosocial.

Específicamente para el área vocacional se distingue una serie de actividades, cuestionarios, escalas y estudios de caso cuyos elementos e indicadores no son pertinentes ni del todo compatibles con las competencias a desarrollar dictadas por los programas de estudio vigentes.

Por su parte, el Manual para apoyar la orientación educativa en planteles de educación media superior (2014) solo establece información acerca de los conceptos y funciones de la orientación tanto vocacional como educativa, soslayando cualquier metodología para su desarrollo.

Aun cuando se pueden localizar algunos instrumentos que incluyen las dimensiones de los programas y planes de estudio, como el caso del Test Conalep-Orienta (Becerril, 2014), sus indicadores no son los mismos que los que contemplan los documentos de la Dirección General del Bachillerato (SEP, 2017), y, por lo tanto, no se constituyen como materiales pertinentes para su aplicación al bachillerato general.

Asimismo, se discute que el uso de instrumentos tales como el Inventario de intereses de Angelini (1984), el test de Kuder (1996) y el test de aptitudes diferenciales [DAT, por su siglas en inglés] (Bennet, 1992), entre otros, que se aplican por uso y costumbre al interior de las instituciones educativas y que no cumplen ni con los perfiles requeridos por los programas de 


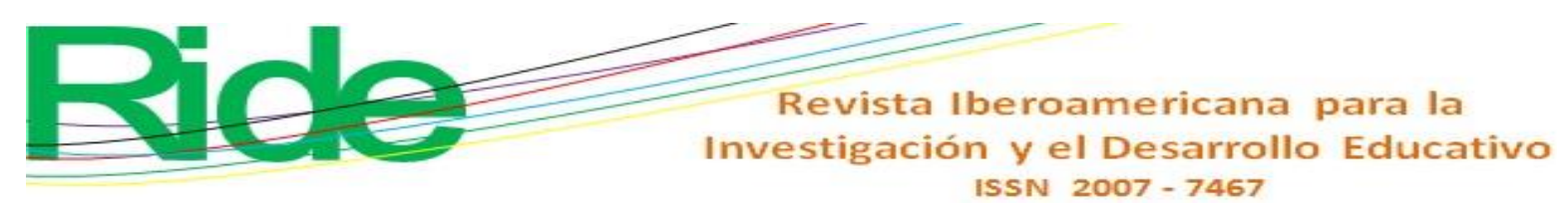

estudio del bachillerato general (SEP, 2017), ni con los indicadores pertinentes a las circunstancias particulares de los estudiantes de nuestro país.

Para subsanar las ausencias anteriores, se realizó el presente estudio con el objetivo de desarrollar y validar un instrumento para la orientación vocacional propedéutica en México.

\section{Materiales y métodos}

\section{Participantes}

Participaron voluntariamente un total de 56 personas:

- 40 estudiantes de cuarto semestre de nivel bachillerato de la ciudad de Xalapa, Veracruz: 20 hombres y 20 mujeres.

- Seis profesionales de la psicología y la educación con experiencia en los campos de evaluación y orientación profesional, dos hombres y cuatro mujeres.

- $\quad 10$ estudiantes de bachillerato para la aplicación del instrumento piloto.

\section{Materiales}

- 10 copias de la escala Ortiz-Barradas para intereses propedéuticos en México para pilotaje.

- $\quad$ Seis copias de la escala Ortiz-Barradas para intereses propedéuticos en México para juicio de expertos.

- $\quad 40$ copias de la escala Ortiz-Barradas para intereses propedéuticos en México.

- 50 lápices con mina $\mathrm{HB} \mathrm{n.}{ }^{\circ}$ 2, grafito color negro.

- 50 gomas de migajón Strike 20.

- Una laptop con sistema operativo Windows 10 Home y paquetería Microsoft Office 2010.

- $\quad$ Un proyector 3LCD HDMI de 3000 lúmenes.

\section{Procedimiento}




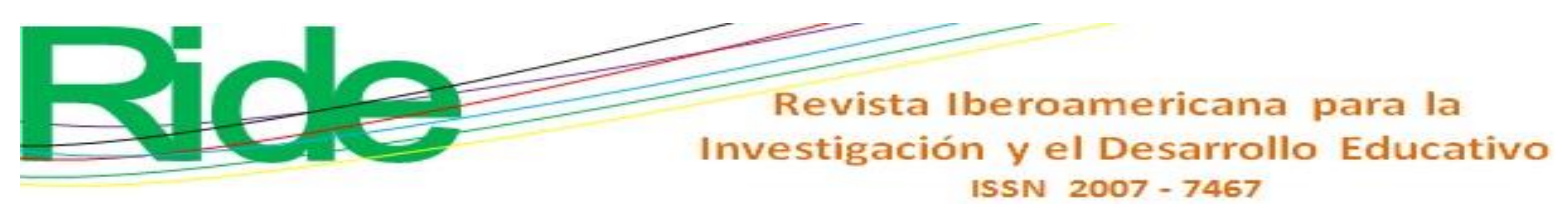

Tabla 1. Diseño del proceso de investigación

\begin{tabular}{|c|c|c|}
\hline Fase 1 & Fase 2 & Fase 3 \\
\hline $\begin{array}{c}\text { Desarrollo, juicio de } \\
\text { expertos y pilotaje del } \\
\text { instrumento }\end{array}$ & Aplicación del instrumento & Análisis de resultados \\
\hline
\end{tabular}

Fuente: Elaboración propia

Como se puede observar en la tabla 1, la investigación fue ordenada en tres fases. En la primera, se consideró el desarrollo del instrumento con base en la revisión documental sobre los indicadores que marcan los programas educativos vigentes y los elementos que deben observar las escalas valorativas de cinco términos.

El Documento base del bachillerato general (Subsecretaría de Educación Media Superior [SEMS], 2017) establece tres tipos de competencias necesarias para la conclusión del programa educativo y la obtención del título de bachiller: genéricas, profesionales básicas y disciplinares.

Dentro de las competencias disciplinares, se distinguen las competencias básicas, que son las "comunes a todos los egresados de la EMS"1 (SEP, 2017, p. 31); y las extendidas, que constituyen el componente de formación propedéutico sobre el cual se sustenta este trabajo.

\section{Diseño del instrumento}

La escala Ortiz-Barradas para intereses propedéuticos en México está compuesta por 50 afirmaciones redactadas en positivo con cinco opciones de respuesta que contemplan los siguientes valores: lo odio (menos dos), me desagrada (menos uno), indeciso (cero), me agrada (uno) y me encanta (dos).

Dichas afirmaciones se asignaron de acuerdo con los elementos descritos por los documentos básicos que establece la SEMS (2017) en cuanto a las competencias que se desarrollan en cada área propedéutica. Así, se constituyeron los siguientes constructos e ítems:

- $\quad$ M: matemáticas:

○ Ítems: 1, 6, 11, 16, 21, 26, 31, 36, 41, 46.

- $\quad$ CE: ciencias experimentales:

○ Ítems: 2, 7, 12, 17, 22, 27, 32, 37, 42, 47.

\footnotetext{
${ }^{1}$ Educación media superior.
} 


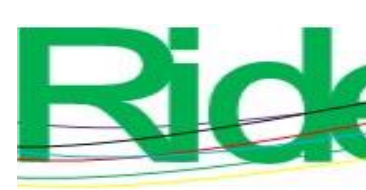

Revista Iberoamericana para la
Investigación y el Desarrollo Educativo
ISSN $2007-7467$

- CS: ciencias sociales:

○ Ítems: $3,8,13,18,23,28,33,38,43,48$.

- H: humanidades:

○ Ítems: 4, 9, 14, 19, 24, 29, 34, 39, 44, 49.

- $\quad$ C: comunicación:

○ Ítems: $5,10,15,20,25,30,35,40,45,50$.

\section{Juicio de expertos}

La validez de contenido del instrumento consistió en tres etapas: selección de expertos, análisis del instrumento y modificación del mismo.

Para la primera, se realizó una búsqueda de especialistas en el tema de acuerdo con los criterios propuestos por Skjong and Wentworth (2000): experiencia, reputación, disponibilidad e imparcialidad.

La segunda consistió en informar a los evaluadores acerca del propósito del ejercicio de validación, así como del objetivo del instrumento, de los atributos, dimensiones e indicadores de la orientación vocacional utilizados para la redacción de los ítems de la escala. Todo esto se les facilitó también en una tabla impresa para su análisis y comparación con el instrumento.

En la tercera y última etapa, se socializaron los comentarios, sugerencias y recomendaciones de los expertos con el autor del presente texto para realizar las modificaciones pertinentes.

\section{Pilotaje}

El apartado del pilotaje del instrumento comprendió la aplicación a un grupo de 10 estudiantes de cuarto semestre de bachillerato de la ciudad de Xalapa, Veracruz, no considerados posteriormente para la aplicación, y cuyos resultados se reportan en este texto.

A este grupo se le pidió que contestara la escala con la indicación de escribir, en la parte reversa de la hoja, todas aquellas observaciones en cuanto los siguientes considerandos:

- Claridad en las afirmaciones que se leen en la proyección de las diapositivas.

- $\quad$ El uso correcto del lenguaje, es decir, si existen algunas palabras o expresiones que no se ajustan a los usos lingüísticos del contexto. 


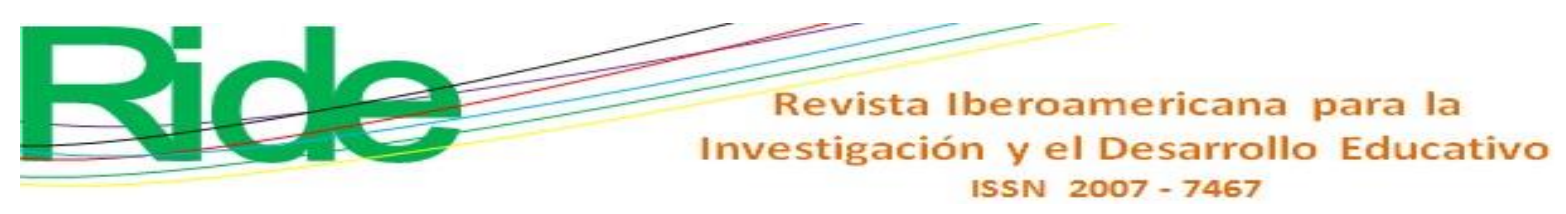

- Diseño amigable del instrumento, esto es, que sean claros los campos a contestar y la suficiencia de los espacios para las respuestas.

Se modificaron los reactivos 2, 5, 25, 34 y 47 en cuanto a redacción, pero no en contenido.

La fase dos consistió en la aplicación de las escalas a cuarenta estudiantes de cuarto semestre de un bachillerato de la ciudad de Xalapa, Veracruz.

Para ello, fue necesaria la gestión del espacio y el permiso de los padres y/o tutores de cada estudiante bajo la lectura y aceptación del consentimiento informado, en donde se les explicó la finalidad, características del instrumento, protección de datos y beneficios del estudio.

El ejercicio consistió en una aplicación grupal guiada a todos los participantes. En esta debieron leer cada afirmación proyectada en unas diapositivas y marcar con una $x$ el campo que expresaba lo más cercano a su opinión y/o percepción acerca de ellas.

Antes de iniciar la tarea se les indicó que la prueba no consistía en un examen de conocimientos y que no repercutía en ningún grado en sus calificaciones de desempeño académico. Asimismo, se les sugirió contestar cada afirmación lo más honestamente posible, que seleccionaran la que más se apegaba a sus gustos y/o intereses personales.

Aplicados todos los instrumentos, se procedió al análisis de los resultados descrito en la tabla 1 como la fase tres de la investigación. Para ello se generó una matriz de datos en el programa Microsoft Excel 2010, en donde se incluyó la codificación de cada estudiante para proteger su identidad personal, así como los cincuenta ítems con sus cinco opciones de respuesta. Posteriormente se trasladaron los datos al programa IBM SPSS Statistics for Windows, Version 23.0 (IBM Corp, 2015).

\section{Resultados}

Para determinar la fiabilidad del instrumento se utilizó el método de consistencia interna basado en el alfa de Cronbach, el cual asume que los ítems (evaluados en escala tipo Likert) miden un mismo constructo y que están altamente correlacionados (Welch y Corner, 1988).

Usando el paquete estadístico para realizar el análisis de consistencia o alfa de Cronbach, se obtuvieron los siguientes resultados (ver tabla 2). 


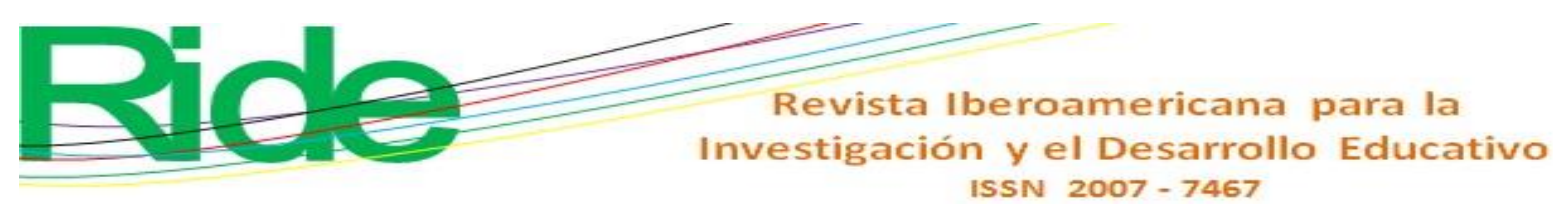

Tabla 2. Estadísticos de fiabilidad

\begin{tabular}{|c|c|c|}
\hline Alfa de Cronbach & $\begin{array}{c}\text { Alfa de Cronbach } \\
\text { basada en los elementos } \\
\text { tipificados }\end{array}$ & Núm. de elementos \\
\hline $\mathbf{8 7 0}$ & .884 & 40 \\
\hline
\end{tabular}

Fuente: Elaboración propia

En la tabla 2 se puede observar que el valor del alfa es de 0.870. Asumiendo las recomendaciones descritas por George y Mallery (2003), quienes sugieren que los coeficientes de alfa de Cronbach ubicados en el rango ocho son buenos y en el nueve excelentes, se puede decir que el cuestionario, debido a los ítems en conjunto, tiene una estabilidad y consistencia fiable.

\section{Discusión}

Los resultados derivados de la presente investigación sugieren la posibilidad del trabajo de la orientación vocacional propedéutica con instrumentos propios y pertinentes a los planes y programas de estudio vigentes al 2017.

Esto hace posible poder generar, en un futuro, manuales de procedimientos específicos para esta actividad educativa que no dependan del uso de instrumentos cuyos indicadores no son compatibles con los del contexto mexicano.

Aunado a ello, se abre la oportunidad para el desarrollo de metodologías para la evaluación propedéutica que coadyuven al proceso de la orientación como un ejercicio integral en donde docentes y alumnos realicen un análisis de sus intereses, aptitudes y competencias que permitan una elección informada, dirigida y confiable de sus áreas de desarrollo personal y profesional.

Aun cuando los estudios en este rubro son incipientes, la información aquí expresada puede constituirse como una línea de investigación cuyos resultados comprensivos den lugar al desarrollo de nuevas formas de diagnóstico, evaluación y planeación en el campo de la psicología educativa.

Sin embargo, esta actividad podría tener algunos problemas inadvertidos. En un primer caso, las escalas valorativas, incluso cuando pueden constituirse como técnicas estadísticamente confiables, no observan las tendencias de las personas a elegir distintas opciones interactivas, más bien sus percepciones respecto a cómo actuarían en tal situación.

En un segundo caso, se podría soslayar el hecho que la educación es un fenómeno que ocurre en contextos sociales. Así, las elecciones podrían estar sesgadas por otras variables más que la opción propedéutica misma (por ejemplo, interacciones verbales con otras personas, historias 


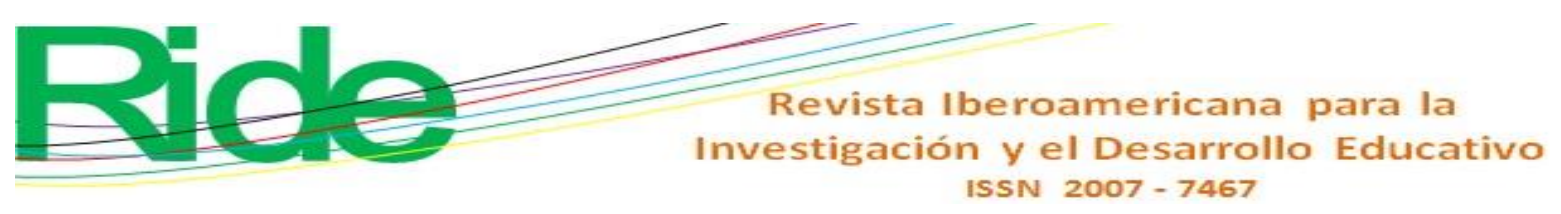

académicas y profesionales de los allegados al estudiante, así como los intereses de los compañeros de salón y otros).

\section{Conclusiones}

Finalmente, para poder subsanar dichas limitaciones, investigaciones futuras en este campo podrían evaluar las preferencias educativas propedéuticas con métodos más experimentales que incluyan sistemáticamente todas las variables antes descritas y con el amparo de una teoría cuyo alcance explicativo pueda abarcar estos fenómenos.

Un fenómeno educativo, al ser social, es testigo de un sistema de relaciones molar y convencional, en donde están involucrados muchos elementos que se interrelacionan entre sí para dar la condición necesaria y suficiente para observarlo.

Uno de los problemas fundamentales de esto se genera al momento de tratar de realizar estudios de corte académico para determinar relaciones, correlaciones y causalidades entre dos o más variables estudiadas. Así, esta consideración no queda fuera del alcance de esta investigación.

Un paso importante del análisis a la síntesis de teorías o modelos académicos se sustenta en la integración teórica de los datos obtenidos con el fin de realizar descripciones cada vez más sistemáticas y empíricas, en vez de causalidades hipotéticas en su constructo. 


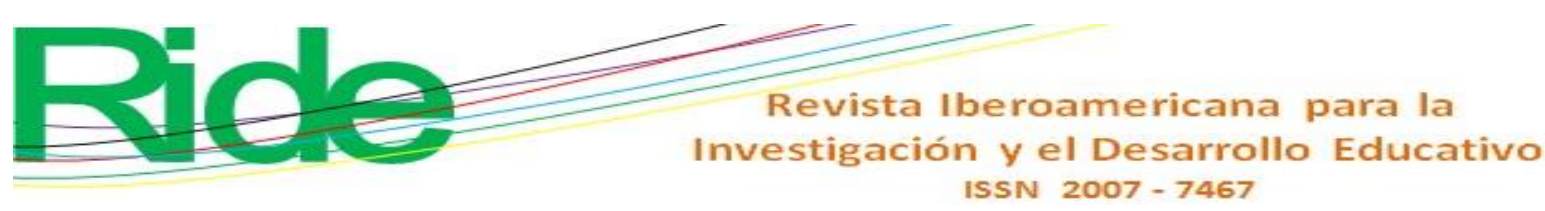

Skjong, R. and Wentworth, B. (2000). Expert Judgement and risk perception. Retrieved from http://research.dnv.com/skj/Papers/SkjWen.pdf.

Subsecretaría de Educación Media Superior [SEMS]. (2014). Manual para apoyar la orientación educativa en planteles de educación media superior. México: Subsecretaría de Educación Media Superior. Recuperado de https://www.sev.gob.mx/educacion-media-superior-ysuperior/files/2015/09/Manual-7_Orientacion_Educativa.pdf.

Subsecretaría de Educación Media Superior [SEMS]. (2017). Documento base del bachillerato general. México: Subsecretaría de Educación Media Superior. Recuperado de https://www.dgb.sep.gob.mx/informacion-academica/programas-deestudio/documentobase/DOC_BASE_16_05_2016.pdf.

Turbay, C. (2000). El derecho a la educación. Desde el marco de la protección integral de los derechos de la niñez y de la política educativa. Colombia: Unicef Colombia.

Trilla, J. (1988). Animación sociocultural, educación y educación no formal. Educar, 13, 17-41.

Welch, S. and Comer, J. (1988). Quantitative Methods for Public Administration: Techniques and Applications. United States: Brooks/Cole Publishing Co. 